\title{
Stochastic Analysis of Project Issues and Fixing its Cause by Project Team and Funding System using Matrix Analytic Method
}

\author{
Rama Ganesan, \\ Independent Researcher \\ B.Tech, Vellore Institute of Technology, \\ Vellore, India
}

\author{
Ramanarayanan. $R$ \\ Professor of Mathematics (Retired), \\ Veltech group of Institutions, \\ Chennai, India
}

\begin{abstract}
Two models in this paper with infinite and finite recording of project issues that come up during implementation are treated. In the models when project team and funding are available, issues come up and they are settled once the cause is fixed. In one model infinite number of issues are recorded. In another model the recording is stopped at a level and it is resumed when the recorded number falls due to settlement. Stationary probabilities of number of issues to be fixed and mean are obtained without the use of iterative methods. Matrix analytic method is used and the matrix $G$ has explicit structure. With the same set of all parameters and by varying the issue rate numerical results of both models for comparison are presented.
\end{abstract}

\section{General Terms}

Project Issues, Fixing, Funding, Manpower.

\section{Keywords}

Infinitesimal generator, Stationary Probability, Matrix Analytic Methods

\section{INTRODUCTION}

In this paper models with project team, its funding system, project issues and fixing them are studied with the application of matrix analytic methods. The models considered here are somewhat different from Queueing theory models. The recording work of issues is stopped in these models when project or funding system is unavailable or capacity is full. In queueing systems, the arrival process is operative and in general, without a break. Since the issues arising in a project wait for fixing the cause, there is a close connection with queueing systems and matrix analytic methods in particular which has not been studied or identified so far at any depth in models with project issues. Aissani.A and Artalejo.J.R [1] and Ayyappan, Subramanian and Gopal Sekar [2] have analyzed retrial queueing systems. Chakravarthy and Neuts [3] have discussed in depth a multi-server queueing model. Daniel and Ramanarayanan [4] have considered (s, S) inventory system with rest periods. Gaver, Jacob and Latouche, [5] have studied finite birth-and-death models in randomly changing environments. Matrix analytic methods have been studied by Latouche and Ramaswami in [6]. Since a project team is essentially a manpower system our models have also a close link with manpower models. Matrix models of manpower system in various environments have been treated by Mohan and Ramanarayanan [7]. For one and two ordering level inventory systems one may refer Murthy and Ramanarayanan [8]. Neuts [9] has presented Matrix analytic solutions in stochastic models and queueing systems. Sales time depending on production has been discussed by Usha, Nithyapriya and Ramanarayanan [10].
Two models (A) and (B) are studied here. In the Models (A) and $(B)$, the situation, when both the project team and the funds are available, is considered as the working / operating state. When the project team has shortage of manpower or the funds for the project are not available, the system is considered as in failed state. When the system is working the project issues come up and the team fixes the cause for them. In Model (A) there is no upper limit for the number of issues to be recorded. In Model (B) there is an upper limit for the number of issues to be recorded. The recording is stopped when the upper limit is reached by the number of issues recorded. The recording starts only after an issue is settled by fixing the cause for it. In matrix analytic models, the results are generated by a matrix $G$ calculated using iterative methods. The matrix $\mathrm{G}$, which comes up here, does not require any iterative procedure. All probabilities, generating function and mean are obtained without using any iterative method. The stationary probability of the number of issues that are waiting for fixing and the expected number of issues to be fixed are derived. With the same set of all parameters and by varying only the issue occurrence and fixing parameters in the models, numerical results are presented for comparison and to discuss the suitability of them.

\section{MODEL (A). RECORDING INFINITE NUMBER OF ISSUES}

\subsection{Assumptions}

i) Finance is available for the project for a random time which has exponential distribution with parameter a. It becomes unavailable for a random time which has exponential distribution with parameter $b$. It alternates between available and unavailable states for the project.

ii) In the project team manpower is available for a random time whose distribution is exponential with parameter $\lambda$ and after which there is shortage of manpower for a random time whose distribution is exponential with parameter $\mu$. There after it again becomes available, unavailable and so on. It alternates in the project team.

iii) When both the finance and the manpower ( $\mathrm{F}$ and $\mathrm{M})$ are available, the time between two epochs of recording issues has exponential distribution with parameter $\mathrm{c}$ and the time between consecutive fixing the cause (settling an issue) is also exponential with parameter $d$. When the manpower is not available or finance is not available or both are not available further recording and fixing of the issues are suspended and stopped. The recording and the fixing of issues begin when both $\mathrm{F}$ and $\mathrm{M}$ become available.

iv) At any recording epoch, the $\mathrm{F}$ and $\mathrm{M}$ system records $\mathrm{N}$ issues at a time with probability $\mathrm{P}(\mathrm{N}=\mathrm{i})=\mathrm{pi}$, for $1 \leq \mathrm{i}<\infty$ where $\sum_{1}^{\infty} p_{i}=1$. 
v) Only one issue is fixed (settled) at a time.

vi) The available time and unavailable time of $F$ and $M$ system and the recording and fixing times of the issues are all independent

\subsection{Analysis}

For studying the above model, the state of the system of the continuous time Markov chain $X$ (t) is as follows. $X(t)=\{(n, i, j): 0 \leq n<\infty$; for $i=0,1$; for $j=0,1\}$. (1) The three co-ordinates $(\mathrm{n}, \mathrm{i}, \mathrm{j})$ represent respectively the number of issues waiting for fixing is $\mathrm{n}$, the state of the manpower system is $i$ and the state of the finance is $j$ for $i, j$ $=0,1$ where the state 0 indicates the unavailable state and the state 1 indicates the available state of the respective manpower system or finance system as the case may be. Let the probability generating function of $\mathrm{N}$, the number of issues recorded at recording epoch be $\Phi(\mathrm{r})=\sum_{i=1}^{\infty} p_{i} r^{i}$ (2) The survivor probabilities are $\mathrm{P}(\mathrm{N}>0)=P_{0}=1$ and $\mathrm{P}(\mathrm{N}>\mathrm{i})=P_{i}=1-\sum_{j=1}^{i} p_{j}$

$$
\text { for } \mathrm{i}=1,2 \ldots . \quad \text { Let } \quad \varnothing(\mathrm{r})=\sum_{i=0}^{\infty} P_{i} r^{i} \text {. }
$$

The relation between the two is $\emptyset(\mathrm{r})=\frac{1-\phi(r)}{1-r}$. infinitesimal generator $Q$ of infinite order which can be presented in block partitioned form with each block is of order 4. The infinitesimal generator of the Model (A) is given below.

$\mathrm{Q}=\left[\begin{array}{ccccccccc}B_{1} & A_{2} & A_{3} & A_{4} & A_{5} & A_{6} & \cdots & A_{j} & \cdots \\ A_{0} & A_{1} & A_{2} & A_{3} & A_{4} & A_{5} & \cdots & A_{j-1} & \cdots \\ 0 & A_{0} & A_{1} & A_{2} & A_{3} & A_{4} & \cdots & A_{j-2} & \cdots \\ 0 & 0 & A_{0} & A_{1} & A_{2} & A_{3} & \cdots & A_{j-3} & \cdots \\ 0 & 0 & 0 & A_{0} & A_{1} & A_{2} & \cdots & A_{j-4} & \cdots \\ 0 & 0 & 0 & 0 & A_{0} & A_{1} & \cdots & A_{j-5} & \cdots \\ 0 & 0 & 0 & 0 & 0 & A_{0} & \cdots & A_{5} & \cdots \\ 0 & 0 & 0 & 0 & 0 & 0 & \cdots & \vdots & \cdots \\ \vdots & \vdots & \vdots & \vdots & \vdots & \ddots & \ddots & \vdots & \ddots\end{array}\right]$

In equation (6) the status of the matrices are listed lexicographically, $\underline{0}, \underline{1}, \underline{2}, \underline{3}, \ldots . \underline{n}, \ldots$ where

$\underline{n}=((\mathrm{n}, 0,0),(\mathrm{n}, 0,1),(\mathrm{n}, 1,0),(\mathrm{n}, 1,1))$. The matrices are all of order 4 . The matrices $B_{1}$ and $A_{1}$ have negative diagonal elements and their off diagonal elements are non- negative. The matrices $A_{0}, A_{2}, A_{3}, \ldots, A_{k+1} \ldots$ have only one positive element in the last row-last column and their other elements are $=0$. They are given as, $A_{0}=$ $\operatorname{diag}(0,0,0, d)$ and $A_{j}=\operatorname{diag}\left(0,0,0, c p_{j-1}\right), 2 \leq \mathrm{j}<\infty$.

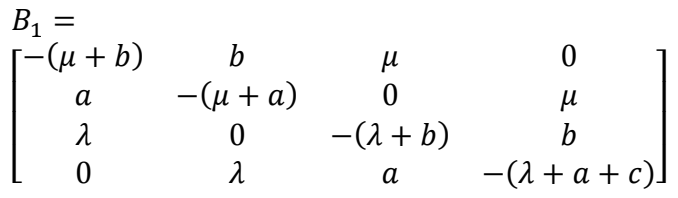

$A_{1}=B_{1}-A_{0}$.

Let $\mathrm{Q}^{\prime}=\sum_{i=0}^{\infty} A_{i}$

This is also an infinitesimal generator of a continuous time Markov chain with finite state space of order 4 . The finite generator matrix $Q^{\prime}$ is given by

$Q^{\prime}=\left[\begin{array}{cccc}-(\mu+b) & b & \mu & 0 \\ a & -(\mu+a) & 0 & \mu \\ \lambda & 0 & -(\lambda+b) & b \\ 0 & \lambda & a & -(\lambda+a)\end{array}\right]$

The probability vector of $\mathrm{w}$ of $\mathrm{Q}$ satisfies $w Q^{\prime}=0 \quad$ and $\quad$ w.e $=1$. (12)
This gives, $\mathrm{w}=\frac{1}{(a+b)(\lambda+\mu)}(\mathrm{a} \lambda, \mathrm{b} \lambda, \mathrm{a} \mu, \mathrm{b} \mu)$.

The stability condition for the existence of a stationary distribution for continuous time Markov chain given by $\mathrm{Q}$ is, (see Neuts [9]) $\quad w A_{0} \mathrm{e}<\mathrm{w}\left[\sum_{j=2}^{\infty}(j-1) A_{j}\right] \mathrm{e} \quad$ (14) This gives, $\mathrm{c} \mathrm{E}(\mathrm{N})<\mathrm{d}$.

Here $\mathrm{E}(\mathrm{N})$ is the expected number of issues recorded at a recording epoch. When the inequality given by (14) is satisfied, the stationary distribution of the process exists, Neuts [9]. Let $\pi(n, i, j)$, for $n=0,1,2,3 \ldots ; i=0,1 ; j=0,1$ be the stationary probability of the states listed in (1) and let $\pi_{k}$ be the vector of type $1 \mathrm{x} 4$ given by,

$\pi_{k}=(\pi(\mathrm{k}, 0,0), \pi(\mathrm{k}, 0,1), \pi(\mathrm{k}, 1,0), \pi(\mathrm{k}, 1,1)), \mathrm{k} \geq 0$.

The stationary probability vector $\pi=\left(\pi_{0}, \pi_{1}, \pi_{3}, \ldots \ldots\right)$ satisfies the equations.

$\pi \mathrm{Q}=0$, and $\pi \mathrm{e}=1$.

The analysis of M/G/1 type of processes is more complicated than that of $\mathrm{G} / \mathrm{M} / 1$ type processes since there is no matrix geometric relationship for the steady state probability vectors. The method of finding the steady state probability vector requires the computation of a stochastic matrix $G$ which satisfies the equation

$\sum_{i=0}^{\infty} A_{i} G^{i}=0$.

The matrix $\mathrm{G}$ is the minimal non-negative solution of $\sum_{i=0}^{\infty} A_{i} X^{i}=0$. Usually it can be found by means of the iteration for convergence of $\mathrm{G}$ starting with $G_{0}=0$; $G_{k+1}=-\left(A_{1}^{-1}\right)\left(A_{0}+\sum_{i=2}^{\infty} A_{i} G^{i}\right), k=0,1,2,3 \ldots \ldots$.

The matrix $A_{0}$ and $A_{j}$ for $\mathrm{j} \geq 2$ have structures with only one non-zero positive element in the last row-last column given by (7). The model, because of this structure, does not require numerical computation to determine the elements of the stochastic matrix $G$. The transitions occur from level $n$ to other levels $n-1$ or $n+1$, or $n+2$, etc.., only from the state (n, 1 , 1). This makes clear that the stochastic matrix $G$ has unit last column vector (all the four elements in the last column $=1$ ) and its all other elements are $=0$. This can be proved also directly. By taking $G_{0}=0$, in (19) it may be seen that the matrix $G$ has only non-zero elements in the last column. Noting this the following equation, (since $\left(-A_{1}^{-1}\right)_{(i, 4)}=\frac{1}{c+d}$, for $\mathrm{i}=1,2,3,4)$, by fixing the unknown last column of $\mathrm{G}$ as $\left(r_{1}, r_{2}, r_{3}, r_{4}\right)$ 'is obtained.

$$
\begin{aligned}
& \mathrm{G}=\left[\begin{array}{llll}
0 & 0 & 0 & r_{1} \\
0 & 0 & 0 & r_{2} \\
0 & 0 & 0 & r_{3} \\
0 & 0 & 0 & r_{4}
\end{array}\right]=\left[\begin{array}{llll}
0 & 0 & 0 & \frac{d}{c+d} \\
0 & 0 & 0 & \frac{d}{c+d} \\
0 & 0 & 0 & \frac{d}{c+d} \\
0 & 0 & 0 & \frac{d}{c+d}
\end{array}\right]+ \\
& {\left[\begin{array}{llll}
0 & 0 & 0 & \frac{c}{c+d} r_{4} \phi\left(r_{4}\right) \\
0 & 0 & 0 & \frac{c}{c+d} r_{4} \phi\left(r_{4}\right) \\
0 & 0 & 0 & \frac{c}{c+d} r_{4} \phi\left(r_{4}\right) \\
0 & 0 & 0 & \frac{c}{c+d} r_{4} \phi\left(r_{4}\right)
\end{array}\right] .}
\end{aligned}
$$

The fourth row elements present in the above equation gives $r_{4}=1$. Substituting it in other rows it can be seen that, $r_{i}=1$ for all $\mathrm{i}=1,2,3,4$. The matrix $\mathrm{G}$ is

$\mathrm{G}=\left[\begin{array}{llll}0 & 0 & 0 & 1 \\ 0 & 0 & 0 & 1 \\ 0 & 0 & 0 & 1 \\ 0 & 0 & 0 & 1\end{array}\right]$.

Equations (16) and (17) present 


$$
\pi_{0} B_{1}+\pi_{1} A_{0}=0
$$

and equation (23). The matrix appearing as the multiplier of the second term of equation (23) is a block Toeplitz matrix. Let it be $\mathrm{D}$ which can be decomposed as $\mathrm{D}=\mathrm{U} \mathrm{L}$ of block upper triangular matrix $\mathrm{U}$ and block lower triangular matrix $\mathrm{L}$ which are presented in (24).

$\pi_{0}\left(A_{2}, A_{3}, A_{4}, \cdots\right)+\left(\pi_{1}, \pi_{2}, \pi_{3}, \ldots\right) x$
$\left[\begin{array}{cccccccc}A_{1} & A_{2} & A_{3} & A_{4} & A_{5} & \cdots & A_{j} & \cdots \\ A_{0} & A_{1} & A_{2} & A_{3} & A_{4} & \cdots & A_{j-1} & \cdots \\ 0 & A_{0} & A_{1} & A_{2} & A_{3} & \cdots & A_{j-2} & \cdots \\ 0 & 0 & A_{0} & A_{1} & A_{2} & \cdots & A_{j-3} & \cdots \\ 0 & 0 & 0 & A_{0} & A_{1} & \cdots & A_{j-4} & \cdots \\ 0 & 0 & 0 & 0 & A_{0} & \cdots & A_{j-5} & \cdots \\ 0 & 0 & 0 & 0 & 0 & \cdots & . & \cdots \\ \vdots & \vdots & \vdots & \vdots & \ddots & \ddots & \vdots & \ddots\end{array}\right]=0$.

$\mathrm{U}=\left[\begin{array}{ccccc}A_{1}^{*} & A_{2}^{*} & A_{3}^{*} & A_{4}^{*} & \cdots \\ 0 & A_{1}^{*} & A_{2}^{*} & A_{3}^{*} & \cdots \\ 0 & 0 & A_{1}^{*} & A_{2}^{*} & \cdots \\ 0 & 0 & 0 & A_{1}^{*} & \cdots \\ \vdots & \vdots & \vdots & \vdots & \ddots\end{array}\right]$ and

$\mathrm{L}=\left[\begin{array}{ccccc}I & 0 & 0 & 0 & \cdots \\ -G & I & 0 & 0 & \cdots \\ 0 & -G & I & 0 & \cdots \\ 0 & 0 & -G & I & \cdots \\ \vdots & \vdots & \vdots & \vdots & \ddots\end{array}\right]$

The matrix $\mathrm{L}$ is known since matrix $\mathrm{G}$ is seen in (21). The inverse of $\mathrm{L}$ can be written in terms of powers of $\mathrm{G}$ as follows.

$L^{-1}=\left[\begin{array}{ccccc}I & 0 & 0 & 0 & \cdots \\ G & I & 0 & 0 & \cdots \\ G^{2} & G & I & 0 & \cdots \\ G^{3} & G^{2} & G & I & \cdots \\ \vdots & \vdots & \vdots & \vdots & \ddots\end{array}\right]$ and $L^{-1} L=I$

From equation (23)

$\pi_{0}\left(A_{2}, A_{3}, A_{4}, \ldots.\right)+\left(\pi_{1}, \pi_{2}, \pi_{3}, \ldots.\right) \mathrm{UL}=0$. This gives $\pi_{0}\left(-A_{2},-A_{3},-A_{4}, \ldots\right) L^{-1}=\left(\pi_{1}, \pi_{2}, \pi_{3}, \ldots\right) \mathrm{U}$ and by writing, $\pi_{0}\left(B_{2}^{*}, B_{3}^{*}, B_{4}^{*} \ldots.\right)=\left(\pi_{1}, \pi_{2}, \pi_{3}, \ldots.\right) \mathrm{U}$, it is noted $B_{i}^{*}=-\sum_{k=i}^{\infty} A_{k} G^{k-i}$ for $\quad \mathrm{i} \geq 2$.

From equation (26)

$\pi_{0}\left(B_{2}^{*}, B_{3}^{*}, B_{4}^{*} \ldots.\right)=\left(\pi_{1}, \pi_{2}, \pi_{3}, \ldots.\right) \mathrm{x}$

$\left[\begin{array}{ccccc}A_{1}^{*} & A_{2}^{*} & A_{3}^{*} & A_{4}^{*} & \cdots \\ 0 & A_{1}^{*} & A_{2}^{*} & A_{3}^{*} & \cdots \\ 0 & 0 & A_{1}^{*} & A_{2}^{*} & \cdots \\ 0 & 0 & 0 & A_{1}^{*} & \cdots \\ \vdots & \vdots & \vdots & \vdots & \ddots\end{array}\right]$

From the above, the following can be seen.

$\pi_{0} B_{2}^{*}=\pi_{1} A_{1}^{*} \Rightarrow \pi_{1}=\pi_{0} B_{2}^{*} A_{1}^{*-1}$

$\pi_{0} B_{3}^{*}=\pi_{1} A_{2}^{*}+\pi_{2} A_{1}^{*}$

$\Longrightarrow \pi_{2}=\pi_{0} B_{3}^{*} A_{1}^{*-1}-\pi_{1} A_{2}^{*} A_{1}^{*-1}$

$\pi_{0} B_{4}^{*}=\pi_{1} A_{3}^{*}+\pi_{2} A_{2}^{*}+\pi_{3} A_{1}^{*}$

$\Longrightarrow \quad \pi_{3}=\pi_{0} B_{4}^{*} A_{1}^{*-1}-\pi_{1} A_{3}^{*} A_{1}^{*-1}-\pi_{2} A_{2}^{*} A_{1}^{*-1}$.

In this manner it can be seen

$\pi_{i}=\pi_{0} B_{i+1}^{*} A_{1}^{*-1}-\sum_{k=1}^{i-1} \pi_{k} A_{i-k+1}^{*} A_{1}^{*-1},$.

for $\mathrm{i} \geq 1$. Now the equation (17), $\pi \mathrm{Q}=0$ reduces to

$$
\begin{aligned}
& \left(\pi_{0}, \pi_{1}, \pi_{2}, \ldots\right) \mathrm{x} \\
& {\left[\begin{array}{ccccccc}
B_{1} & -B_{2}^{*} & -B_{3}^{*} & -B_{4}^{*} & \cdots & -B_{j+1}^{*} & \cdots \\
A_{0} & A_{1}^{*} & A_{2}^{*} & A_{3}^{*} & \cdots & A_{j}^{*} & \cdots \\
0 & 0 & A_{1}^{*} & A_{2}^{*} & \cdots & A_{j-1}^{*} & \cdots \\
0 & 0 & 0 & A_{1}^{*} & \cdots & A_{j-2}^{*} & \cdots \\
\vdots & \vdots & \vdots & \vdots & \ddots & \vdots & \ddots
\end{array}\right]=0}
\end{aligned}
$$

From (29) and (22), $\pi_{0}\left(B_{1}+B_{2}^{*} A_{1}^{*-1} A_{0}\right)=0$

This gives $\pi_{0}$ up to a multiplicative constant. From equation (17) the normalizing condition $\pi \mathrm{e}=1$ becomes $\pi_{0} \mathrm{e}+\pi_{0}\left(\sum_{i=2}^{\infty} B_{i}^{*}\right)\left(\sum_{i=1}^{\infty} A_{i}^{*}\right)^{-1} \mathrm{e}=1$.

The Toeplitz matrix D (given in (23) as the second vector multiplier) and equation $\mathrm{UL}=\mathrm{D}$ and (24) give, $\mathrm{U}=$ $\mathrm{D} L^{-1}=\left[\begin{array}{cccc}A_{1} & A_{2} & A_{3} & \cdots \\ A_{0} & A_{1} & A_{2} & \cdots \\ 0 & A_{0} & A_{1} & \cdots \\ 0 & 0 & A_{0} & \cdots \\ \vdots & \vdots & \vdots & \ddots\end{array}\right]\left[\begin{array}{ccccc}I & 0 & 0 & 0 & \cdots \\ G & I & 0 & 0 & \cdots \\ G^{2} & G & I & 0 & \cdots \\ G^{3} & G^{2} & G & I & \cdots \\ \vdots & \vdots & \vdots & \vdots & \ddots\end{array}\right]$.

$A_{1}^{*}=\sum_{k=1}^{\infty} A_{k} G^{k-1}$ and $A_{i}^{*}=\sum_{k=i}^{\infty} A_{k} G^{k-i}$ for $\mathrm{i} \geq 2$.

Now $\mathrm{G}=G^{k}$ for $\mathrm{k} \geq 1$ from (21).Using (7),(27), (37) and (3) it is noted for $\mathrm{i} \geq 2, \quad A_{i}^{*}=-B_{i}^{*}=\operatorname{diag}\left(0,0,0, \mathrm{c} P_{i-2}\right)$ and $A_{1}^{*}=\left[\begin{array}{cccc}-(\mu+b) & b & \mu & 0 \\ a & -(\mu+a) & 0 & \mu \\ \lambda & 0 & -(\lambda+b) & b \\ 0 & \lambda & a & -(\lambda+a+d)\end{array}\right]$

Now the matrix multiplier of $\pi_{0}$ in equation (34) becomes $B_{1}+B_{2}^{*} A_{1}^{*-1} A_{0}=B_{1}+\operatorname{diag}(0,0,0,-\mathrm{c}) A_{1}^{*-1} \operatorname{diag}(0,0,0, \mathrm{~d})$. Because of the diagonal structure of the matrices the last row of the matrix $A_{1}^{*-1}$ is needed. The last row of matrix $A_{1}^{*-1}$ may be seen using the cofactor ad-joint method. $\left(A_{1}^{*-1}\right)_{(r 0 w 4)}=$ $(-\lambda \mathrm{a},-\lambda \mathrm{b},-\mathrm{a} \mu,-\mathrm{b} \mu)\left(\frac{1}{d b \mu}\right)$ $B_{1}+B_{2}^{*} A_{1}^{*-1} A_{0}=$

$$
\left[\begin{array}{cccc}
-(\mu+b) & b & \mu & 0 \\
a & -(\mu+a) & 0 & \mu \\
\lambda & 0 & -(\lambda+b) & b \\
0 & \lambda & a & -(\lambda+a)
\end{array}\right]
$$

This is the basic generator Q' given in (11). Taking the normalizing condition from (35) it is noted that $\sum_{i=2}^{\infty} B_{i}^{*}=\operatorname{diag}\left(0,0,0,-\mathrm{c} \sum_{i=2}^{\infty} P_{i-2}\right)$

$=\operatorname{diag}(0,0,0,-\mathrm{c} \mathrm{E}(\mathrm{N}))$.

This is obtained from (5) since $\sum_{i=2}^{\infty} P_{i-2}=$

$\lim _{r \rightarrow 1} \emptyset(\mathrm{r})=\lim _{r \rightarrow 1} \frac{1-\phi(r)}{1-r}=\phi^{\prime}(1)=\mathrm{E}(\mathrm{N})$.

From (38) the sum of the matrices, $\sum_{i=1}^{\infty} A_{i}^{*}=$

$\left[\begin{array}{cccc}-(\mu+b) & b & \mu & 0 \\ a & -(\mu+a) & 0 & \mu \\ \lambda & 0 & -(\lambda+b) & b \\ 0 & \lambda & a & -(\lambda+a+d)+c E(N)\end{array}\right]$

(43) $\mathrm{To}$

use the equation (35) of normalizing condition, only the last row elements of the inverse of the above matrix is required because of the diagonal structure of the multiplier matrix given in (41). The last row of matrix $\left(\sum_{i=1}^{\infty} A_{i}^{*}\right)^{-1}$ using the cofactor ad-joint method, is seen as

$(-\lambda \mathrm{a},-\lambda \mathrm{b},-\mathrm{a} \mu,-\mathrm{b} \mu)\left(\frac{1}{b \mu(d-c E(N)}\right)$. So the normalizer is $\pi_{0}\left(1,1,1,1+\frac{c E(N)(\pi+\mu)(a+b)}{b \mu(d-c E(N))}\right)^{\prime}=1$

where' indicates the transpose. The matrix multiplier given in (34) evaluated in (40) may now be used to obtain $\pi_{0}$ explicitly by replacing the first column of the matrix in (40) by the normalizer (44). This gives 
$\pi_{0}\left[\begin{array}{cccc}1 & b & \mu & 0 \\ 1 & -(\mu+a) & 0 & \mu \\ 1 & 0 & -(\lambda+b) & b \\ 1+\frac{c E(N)(\pi+\mu)(a+b)}{b \mu(d-c E(N))} & \lambda & a & -(\lambda+a)\end{array}\right]$
$=(1,0,0,0)$.

The multiplier matrix above of $\pi_{0}$ is invertible and by multiplication of the inverse of it on both sides gives $\pi_{0}=\frac{d-c E(N)}{d(\lambda+\mu)(a+b)}(\lambda a, \lambda b, a \mu, b \mu)$.

Using the equations (29), (30), (31) and the values given in (38) by repeated calculations the probabilities of number of recorded issues waiting for fixing may be obtained as follows. $\pi_{1}=\pi_{0} \frac{c}{d}$ and $\pi_{2}=\pi_{0}\left(\frac{c}{d} P_{1}+\left(\frac{c}{d}\right)^{2}\right)$ with other probabilities as $\pi_{3}=\pi_{0}\left(\frac{c}{d} P_{2}+2 P_{1}\left(\frac{c}{d}\right)^{2}+\left(\frac{c}{d}\right)^{3}\right)$,

$\pi_{4}=\pi_{0}\left(\frac{c}{d} P_{3}+2 P_{2}\left(\frac{c}{d}\right)^{2}+3\left(\frac{c}{d}\right)^{3} P_{1}+\left(\frac{c}{d} P_{1}\right)^{2}+\left(\frac{c}{d}\right)^{4}\right.$

The recursive method, although theoretically possible, may not be suitable for writing the probabilities in acceptable compact closed form. The generating function is presented below by noting the structure of the matrices. The matrix factors appearing as multipliers in equation (32), may be seen as follows. This is due to the diagonal structures presented in equations (38) for $A_{i}^{*}$ and $B_{i}^{*}, \mathrm{i} \geq 2$ and the requirement of only the fourth row of $A_{1}^{*-1}$ given in (39) for calculation. $B_{i+1}^{*} A_{1}^{*-1}=\mathrm{c} P_{i-1} \tilde{A}$ and $A_{i+1}^{*} A_{1}^{*-1}=-\mathrm{c} P_{i-1} \tilde{A}$, for $\mathrm{i} \geq 1$

Here $\tilde{A}==\left[\begin{array}{cccc}0 & 0 & 0 & 0 \\ 0 & 0 & 0 & 0 \\ 0 & 0 & 0 & 0 \\ a \lambda & b \lambda & a \mu & b \mu\end{array}\right]\left(\frac{1}{d b \mu}\right)$

The probabilities of various recorded issue levels are listed.

$\pi_{0}=\pi_{0}$

$\pi_{1}=\pi_{0} \mathrm{c} P_{0} \tilde{A}$

$\pi_{2}=\pi_{0} \mathrm{c} P_{1} \tilde{A}+\pi_{1} \mathrm{c} P_{0} \tilde{A}$

$\pi_{3}=\pi_{0} \mathrm{c} P_{2} \tilde{A}+\pi_{1} \mathrm{c} P_{1} \tilde{A}+\pi_{2} c P_{0} \tilde{A}$

$\pi_{i}=\pi_{0} \mathrm{c} P_{i-1} \tilde{A}+\pi_{1} \mathrm{c} P_{i-2} \tilde{A}+\pi_{2} c P_{i-3} \tilde{A}+\cdots+\pi_{i-1} c P_{0} \tilde{A}$

for $\mathrm{i} \geq 1$.

Multiplying the row $\mathrm{i}$ above by $r^{i}$ for $\mathrm{i} \geq 0$ and finding the sum vertically to get the probability generating function, $\Psi(\mathrm{r})=\sum_{i=1}^{\infty} \pi_{i} r^{i}$ it can be seen,

$\Psi(\mathrm{r})=\pi_{0}+[\mathrm{r} \Psi(\mathrm{r}) \varnothing(\mathrm{r})] \mathrm{c} \tilde{A}$

The function $\Psi$ (r) of the left side also appears on the right side but the matrix $\tilde{A}$ has non-zero elements only in the last row. Only the fourth coordinate of the vector function $\Psi$ (r) is required. Taking the function to the left side and by inversion $\Psi(\mathrm{r})=\pi_{o}(\mathrm{I}-\mathrm{r} \emptyset(\mathrm{r}) \mathrm{c} \tilde{A})^{-1}$.

The matrix $\mathrm{I}-\mathrm{r} \emptyset(\mathrm{r}) \mathrm{c} \tilde{A}=$

$$
\begin{aligned}
& {\left[\begin{array}{cccc}
1 & 0 & 0 & 0 \\
0 & 1 & 0 & 0 \\
0 & 0 & 1 & 0 \\
-a \lambda f(r) & -b \lambda f(r) & -a \mu f(r) & 1-b \mu f(r)
\end{array}\right]} \\
& \text { and } f(r)=r \emptyset(\mathrm{r}) \frac{c}{d b \mu} .
\end{aligned}
$$

Taking the inverse of the matrix given in (53), it is seen

$$
\Psi(\mathrm{r})=\pi_{o}\left[\begin{array}{cccc}
1 & 0 & 0 & 0 \\
0 & 1 & 0 & 0 \\
0 & 0 & 1 & 0 \\
a \lambda f(r) & b \lambda f(r) & a \mu f(r) & 1
\end{array}\right] \frac{1}{(1-b \mu f(r))}
$$

Using the equation (46) the fourth coordinate of vector function $\Psi(\mathrm{r})$ is $\frac{(d-c E(N)) b \mu}{d(\lambda+\mu)(a+b)} \frac{1}{(1-b \mu f(r))}=\frac{(d-c E(N)) b \mu}{d(\lambda+\mu)(a+b)} \frac{1}{\left(1-r \emptyset(r) \frac{c}{d}\right)}$.

Substituting in (51) and simplifying, the probability generating function of the stationary probabilities of number of issues waiting for fixing is seen as,

$$
\Psi(\mathrm{r})=\pi_{0}\left\lceil\frac{1}{\left(1-r \varnothing(r) \frac{c}{d}\right)}\right\rceil .
$$

The vector $\pi_{0}$ is given by (46) and the probability generating function $\varnothing(r)$ is given by (4). The state probabilities given in the recurrence relation (49) can be obtained using Binomial expansion and comparing coefficients of $r$ on both sides here in (57). Binomial expansion is valid due to stability condition (15) that c E (N) < d and $\varnothing(1)=\Phi^{\prime}(1)=\mathrm{E}(\mathrm{N})$ using (42). Using diffenciation, the expected stock of recorded issues waiting for fixing $\mathrm{E}(\mathrm{K})$ is seen as follows.

$\mathrm{E}(\mathrm{K})=\left\lceil\frac{\frac{c}{d}\left(E(N)+\emptyset^{\prime}(1)\right)}{\left(1-E(N) \frac{c}{d}\right)}\right\rceil$.

The probability of no recorded issue waiting for fixing is $\pi_{0} e$ $=\left[1-E(N) \frac{c}{d}\right]$.

\section{MODEL (B). RECORDING ONLY FINITE NUMBER OF ISSUES}

In Model (A) the recording of the number of issues are continued without limitation. Here in Model (B) the recording is stopped at an upper limit. It begins then after next fixing and again it stops at the upper limit and so on. The assumptions (i, ii, iii, v and vi) of Model (A) are unchanged for Model (B) and the assumption (iv) is changed.

\subsection{Revised Assumption for Recording}

(iv). The upper limit of the number of issues recorded is $\mathrm{M}$. At any recording epoch, when the gap is $k$ to reach the upper limit $\mathrm{M}$, for $1 \leq \mathrm{k} \leq \mathrm{M}$, the $\mathrm{F}$ and $\mathrm{M}$ system records $\mathrm{N}$ issues at a time with $\mathrm{P}(\mathrm{N}=\mathrm{i})=\mathrm{pi}$, for $1 \leq \mathrm{i}<\mathrm{k}$ and $\mathrm{P}(\mathrm{N}=\mathrm{k})$ $=P_{k-1}$, where $P_{k-1}=1-\sum_{i=1}^{k-1} p_{i}=\sum_{k}^{\infty} p_{i}$ and $\sum_{1}^{\infty} p_{i}=1$.

\subsection{Analysis}

For studying the above model, the state of the system of the continuous time Markov chain $X(t)$ is defined as follows. $X(t)=\{(n, i, j): 0 \leq n \leq M$; for $i=0,1$; for $j=0,1\}$. (60) The three co-ordinates $(n, i, j)$ represent respectively the number of issues waiting for fixing is $n$, for $0 \leq n \leq M$, the state of the manpower of the project team is $i$ and the state of the finance is $\mathrm{j}$ for $\mathrm{i}, \mathrm{j}=0,1$ where the state 0 indicates the unavailable state and the state 1 indicates the available state of the respective manpower or finance system as the case may be. The continuous time Markov chain describing model has the infinitesimal generator $\mathrm{Q}^{\prime \prime}$ of order $4(\mathrm{M}+1)$ which can be presented in block partitioned form in which each block is of order 4. The infinitesimal generator of the model is given below.

$\mathrm{Q}^{\prime \prime}=\left[\begin{array}{ccccccc}B_{1} & A_{2} & A_{3} & \cdots & A_{M-1} & A_{M} & A_{M+!}^{*} \\ A_{0} & A_{1} & A_{2} & \cdots & A_{M-2} & A_{M-1} & A_{M}^{*} \\ 0 & A_{0} & A_{1} & \cdots & A_{M-3} & A_{M-2} & A_{M-1}^{*} \\ 0 & 0 & A_{0} & \cdots & A_{M-4} & A_{M-3} & A_{M-2}^{*} \\ \vdots & \vdots & \vdots & \ddots & \vdots & \vdots & \vdots \\ 0 & 0 & 0 & \cdots & A_{0} & A_{1} & A_{2}^{*} \\ 0 & 0 & 0 & \cdots & 0 & A_{0} & A_{1}^{*}\end{array}\right]$

In equation (61) the states of the matrices are listed lexicographically as $\underline{0}, \underline{1}, \underline{2}, \underline{3}, \ldots n, \ldots \underline{M}$ where $\underline{n}=((\mathrm{n}, 0,0),(\mathrm{n}, 0,1),(\mathrm{n}, 1,0),(\mathrm{n}, 1,1))$, for $0 \leq \mathrm{n} \leq \mathrm{M}$. The matrices are all of order 4. The matrices, $B_{1}, A_{0}, A_{1}, A_{2}, A_{3}, \ldots$, and $A_{M}$ are as defined already in 
equations (7), (8) and (9). The matrices $A_{i}^{*}$ are as given in equation (38) for $1 \leq \mathrm{i} \leq \mathrm{M}+1$. Let $\pi(n, i, j)$, for $n=0,1,2,3$ ....M ; $\mathrm{i}=0,1 ; \mathrm{j}=0,1$ be the stationary probability of the states listed and let $\pi_{k}$ be the vector of type 1 x 4 given by, $\pi_{k}=(\pi(\mathrm{k}, 0,0), \pi(\mathrm{k}, 0,1), \pi(\mathrm{k}, 1,0), \pi(\mathrm{k}, 1,1)), 0 \leq \mathrm{k} \leq \mathrm{M}(62)$ The stationary probability vector $\pi=\left(\pi_{0}, \pi_{1}, \ldots \ldots \ldots \pi_{M}\right)$ satisfies the equations, $\pi \mathrm{Q}^{\prime \prime}=0$, and $\pi \mathrm{e}=1$. (63)

\section{NUMERICAL RESULTS}

Various values are considered for the parameter $\mathrm{c}$ of recording in the examples of the two models (A) and (B), with same values for all other parameters. The values of are given below in the table 1 and values of $c$ are listed in table 2 . The calculated values of $\mathrm{E}(\mathrm{N})$ and $\Phi^{\prime}(1)$ are also presented in table 1 . The maximum capacity is considered as $\mathrm{M}=5$ for Model (B). Various probabilities of stationary levels for the two models and expected values for different $\mathrm{c}$ values are tabulated. Symbol* is used to indicate the values for Model (B). As c increases, in Figure.1, expected stock of issues
This being a finite system, the stationary probability vector $\pi$ can be obtained easily. Replacing the first column of $\mathrm{Q}^{\prime \prime}$ by e and calling that matrix as $\mathrm{Q}^{\prime \prime \prime}$ and using matrix method of inversion, one can get, $\pi=(1,0,0 \ldots \ldots 0)\left(Q^{\prime \prime \prime}\right)^{-1}$ It is also interesting to note that the decomposition method, applied for Model (A), is applicable here.

waiting for fixing $\mathrm{E}(\mathrm{K})$ values also increase in the two models and the increase is much more in Model (A) compared to Model (B). Significant variations can also be seen in probability values for the two Models in Figure 2. For the set of numerical values, the expected stock of issues waiting for fixing $\mathrm{E}^{*}(\mathrm{~K})$ and the other probabilities show some regularity when $\mathrm{c}$ increases in the Model (B). When $\mathrm{c}$ is small, the fixing (settlement) of issues is more and the probability of issue level is zero is more in the two Models. Table 3 considers the case when both rates $\mathrm{c}$ and $\mathrm{d}$ vary together.

Table.1.The parameter values for the Model (A) and Model (B).

\begin{tabular}{|r|r|r|r|r|r|r|r|r|r|r|r|r|r|}
\hline $\boldsymbol{\lambda}$ & $\boldsymbol{\mu}$ & $\mathbf{a}$ & $\mathbf{b}$ & $\mathbf{p 1}$ & $\mathbf{p 2}$ & $\mathbf{p 3}$ & $\mathbf{p 4}$ & $\mathbf{P 1}$ & $\mathbf{P 2}$ & $\mathbf{P 3}$ & $\mathbf{E}(\mathbf{N})$ & $\boldsymbol{\Phi}^{\prime}(\mathbf{1})$ & $\mathbf{d}$ \\
\hline 1 & 10 & 2 & 20 & 0.4 & 0.3 & 0.2 & 0.1 & 0.6 & 0.3 & 0.1 & 2 & 1.5 & 20 \\
\hline
\end{tabular}

Table.2.The probabilities and expected values for the Model (A) and Model (B).

\begin{tabular}{|l|r|r|r|r|r|r|r|r|}
\hline $\mathbf{C}$ & $\mathbf{2}$ & $\mathbf{3}$ & $\mathbf{4}$ & $\mathbf{5}$ & $\mathbf{6}$ & $\mathbf{7}$ & $\mathbf{8}$ & $\mathbf{9}$ \\
\hline $\mathrm{P}(\mathrm{n}=0)$ & 0.8 & 0.7 & 0.6 & 0.5 & 0.4 & 0.3 & 0.2 & 0.1 \\
\hline $\mathrm{P}(\mathrm{n}=1)$ & 0.08 & 0.105 & 0.12 & 0.125 & 0.12 & 0.105 & 0.08 & 0.045 \\
\hline $\mathrm{P}(\mathrm{n}=2)$ & 0.056 & 0.07875 & 0.096 & 0.10625 & 0.108 & 0.09975 & 0.08 & 0.04725 \\
\hline $\mathrm{P}(\mathrm{n}=3)$ & 0.0344 & 0.0527625 & 0.0696 & 0.0828125 & 0.09 & 0.0884625 & 0.0752 & 0.04691295 \\
\hline $\mathrm{P}(\mathrm{n}=4)$ & 0.0172 & 0.030226875 & 0.04464 & 0.05851625 & 0.06924 & 0.073434375 & 0.06688 & 0.044443125 \\
\hline $\mathrm{P}(\mathrm{n}>4)$ & 0.0124 & 0.033260625 & 0.06976 & 0.127421875 & 0.21276 & 0.333353125 & 0.49792 & 0.716394375 \\
\hline $\mathrm{E}(\mathrm{K})$ & 2.125 & 2.571428571 & 3.166666667 & 4 & 5.25 & 7.3333333333 & 11.5 & 24 \\
\hline $\mathrm{P}(\mathrm{n}=0)$ & 0.804939106 & 0.713455208 & 0.627825213 & 0.549014562 & 0.477593692 & 0.413750427 & 0.357347055 & 0.30800016 \\
\hline $\mathrm{P}(\mathrm{n}=1)$ & 0.080493911 & 0.107018281 & 0.125565043 & 0.13725364 & 0.143278108 & 0.144812649 & 0.142938822 & 0.138600072 \\
\hline $\mathrm{P}^{*}(\mathrm{n}=2)$ & 0.056345737 & 0.080263711 & 0.100452034 & 0.116665594 & 0.128950297 & 0.137572017 & 0.142938822 & 0.145530075 \\
\hline $\mathrm{P}^{*}(\mathrm{n}=3)$ & 0.034612382 & 0.053776686 & 0.072827725 & 0.090930537 & 0.107458581 & 0.122004657 & 0.134362493 & 0.144490575 \\
\hline $\mathrm{P}^{*}(\mathrm{n}=4)$ & 0.017306191 & 0.030807888 & 0.046710196 & 0.06425186 & 0.082671468 & 0.101278347 & 0.119496855 & 0.136884896 \\
\hline $\left.\mathrm{P}^{*}(\mathrm{n}>4)\right)$ & 0.006302673 & 0.014678226 & 0.026619789 & 0.041883806 & 0.060047855 & 0.080581904 & 0.102915952 & 0.126494222 \\
\hline $\mathrm{E}^{*}(\mathrm{~K})$ & 0.397760659 & 0.625498443 & 0.864892014 & 1.109802912 & 1.35447959 & 1.593993559 & 1.824471126 & 2.043142641 \\
\hline
\end{tabular}

Table 3. Expectation and Probability Values when Issue Rates (c) and Fixing Rates (d) vary Together

\begin{tabular}{|c|c|c|c|c|c|c|}
\hline$(\mathbf{c}, \mathbf{d}) \backslash E_{\text {, }}^{*}$ & $\mathbf{E}(\mathbf{K})$ & $\mathbf{E} *(\mathbf{K})$ & $\mathbf{P}(\mathbf{n}=\mathbf{0})$ & $\mathbf{P *}(\mathbf{n}=\mathbf{0})$ & $\mathbf{P}(\mathbf{n}>\mathbf{4})$ & $\mathbf{P}^{*}(\mathbf{n}>\mathbf{4})$ \\
\hline$(3,20)$ & 2.571428571 & 0.625498443 & 0.7 & 0.713455208 & 0.033260625 & 0.014678226 \\
\hline$(3,23)$ & 2.382352941 & 0.534656627 & 0.739130435 & 0.748621756 & 0.023509387 & 0.010970076 \\
\hline$(3,26)$ & 2.25 & 0.466225343 & 0.769230769 & 0.776239655 & 0.01744707 & 0.008494489 \\
\hline$(6,20)$ & 5.25 & 1.35447959 & 0.4 & 0.477593692 & 0.21276 & 0.060047855 \\
\hline$(6,23)$ & 4.227272727 & 1.163198435 & 0.47826087 & 0.532847576 & 0.143374609 & 0.04560295 \\
\hline$(6,26)$ & 3.642857143 & 1.015334481 & 0.538461538 & 0.578474155 & 0.102347418 & 0.035643626 \\
\hline$(9,20)$ & 24 & 2.043142641 & 0.1 & 0.30800016 & 0.716394375 & 0.126494222 \\
\hline
\end{tabular}




\begin{tabular}{|l|c|c|c|c|c|c|}
$(9,23)$ & 10.5 & 1.785172123 & 0.217391304 & 0.366636133 & 0.465722663 & 0.098927267 \\
\hline$(9,26)$ & 7.125 & 1.575847692 & 0.307692308 & 0.418393831 & 0.322633904 & 0.078931162 \\
\hline
\end{tabular}

In table 3 expected values of stocks of issues, probabilities of zero issues and probabilities of higher number of issues obtained from calculations are tabulated when the issue rates and fixing rates (c, d) are $=(3,20),(3,23),(3,26),(6,20)$, $(6,23),(6,26),(9,20),(9,23)$ or $(9,26)$. Both $E(K)$ and $E^{*}(K)$ increase when $\mathrm{c}$ increases from 3 to 9 and they decrease when $\mathrm{d}$ increases. The probability of zero issues increases when $\mathrm{d}$ increases and when $c$ increases it decreases and higher probabilities also increase when $\mathrm{c}$ increases and they decrease

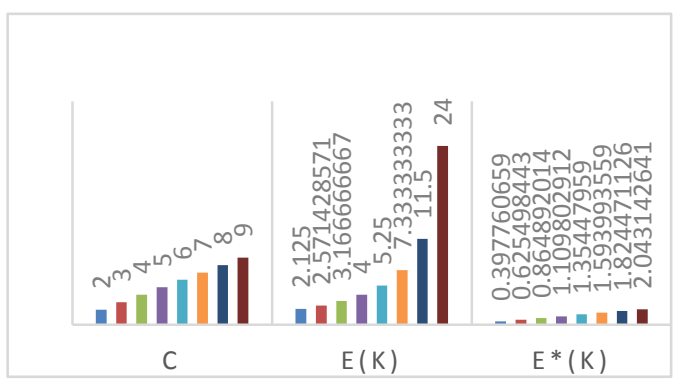

Figure.1 E (Issues) of (A), (B) for c Values

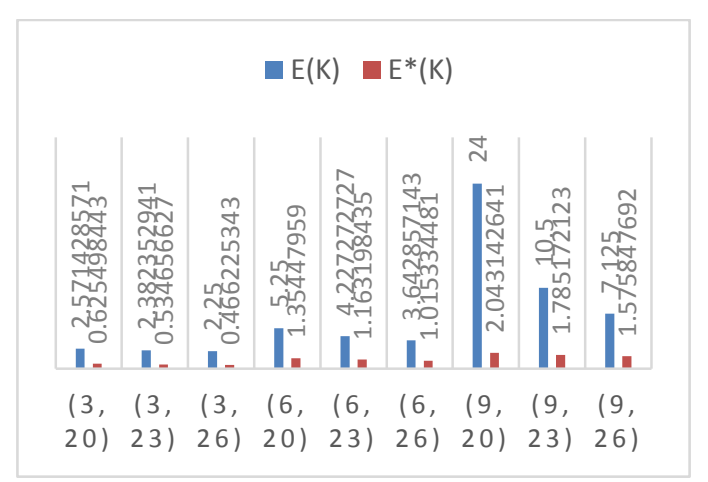

Figure 3. Expectations Rates Vary

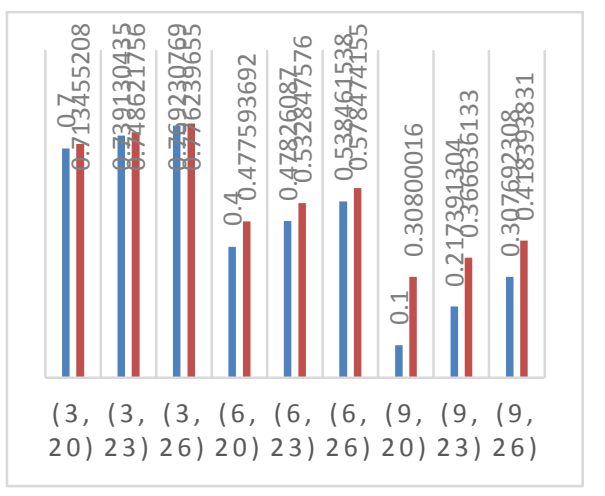

Figure 5.Probability of $n=0$ When Rates Vary.

\section{CONCLUSION}

Two models have been treated in this paper with infinite and finite recording of project issues. In the models when project team and funding are available, issues come up and they are settled once the cause is fixed. In one model infinite numbers of issues are recorded. In another model the recording is stopped at a level and it is resumed when the recorded number falls due to settlement. Matrix analytic methods are used to present results. In the numerical examples it has been noted that stopping at a level is advantageous since the probabilities when $\mathrm{d}$ increases. In the Model (B) when the recording is stopped at a level presents significant results since the expected values do not increase rapidly and the probabilities of lower levels are high compared to probabilities of higher levels. The examples directly indicate for better running of the projects, $\mathrm{F}$ and $\mathrm{M}$ system may have to find ways for improving the fixing rate and may have to find some stopping level for recording issues and to find ways to decrease issue rates so that project issues are fixed early

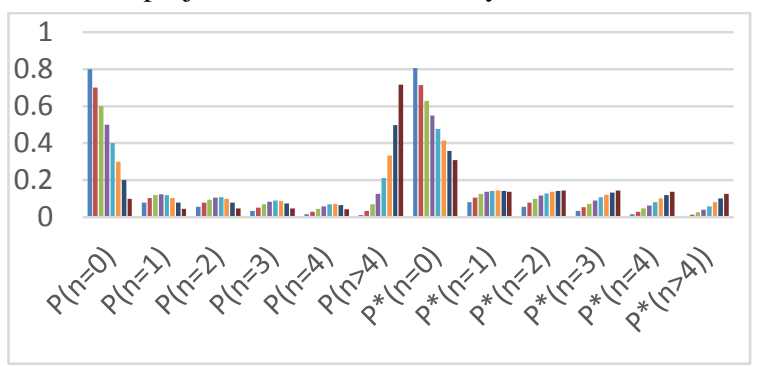

Figure. 2. Probabilities obtained for (A),(B)

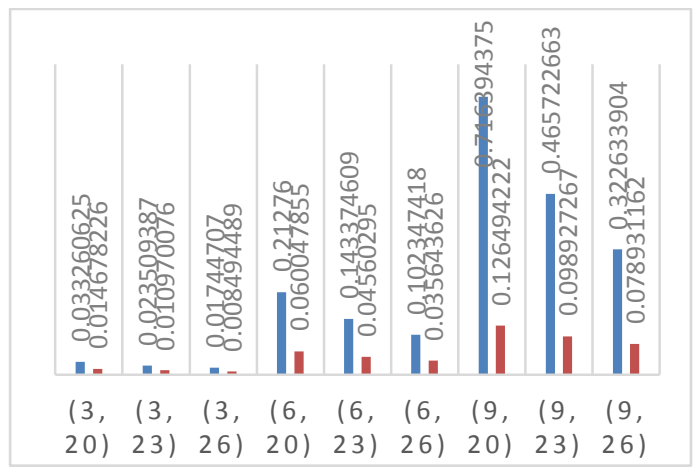

Figure 4. Probabilities for $n>4$ When Rates Vary

are more for lower levels and the expected values are less. Models with randomly varying rates and with phase type arrival and fixing in this area may be other important fields for further research studies.

\section{REFERENCES}

[1] Aissani.A. and Artalejo.J.R. 1998. On the single server retrial queue subject to break downs, Queueing System.30, 309-321.

[2] Ayyappan.G, Muthu Ganapathy Subramanian.A and Gopal Sekar. 2010. M/M/1 retrial queueing system with loss and feedback under pre-emptive priority service, IJCA, Vol.2,N0.6,.27-34.

[3] Chakravarthy.S.R and Neuts. M.F.2014. Analysis of a multiserver queueing model with MAP arrivals of special customers, Simulation Modeling Practice and Theory, vol.43,79-95,

[4] Daniel.J.K, and Ramanarayanan.R, 1988. An (S, s) inventory system with rest periods to the server, Naval Research-Logistics,35,119-123.

[5] Gaver, D., Jacobs, P., Latouche, G, 1984. Finite birth-and-death models in randomly changing environments. Probability, 16,715-731
Advances-in-Applied- 
[6] Latouche.G, and Ramaswami .V, (1998). Introduction to Matrix Analytic Methods in Stochastic Modeling, SIAM, Philadelphia, Mohan C. and Ramanarayanan R, 2010.An analysis of manpower, Money and Business with Random Environments, International Journal of Applied Mathematics,23,(5),pp.927-940.

[7] Murthy.S, and Ramanarayanan.R, 2008.One ordering and two ordering levels Inventory systems units with SCBZ lead times, International Journal of Pure and Applied Mathematics,47,(3),pp.427-447.

[8] Neuts .M.F.1981. Matrix- Geometric Solutions in Stochastic Models: An algorithmic Approach, The Johns Hopkins-Press,-Baltimore

[9] Usha.k, Nithyapriya.N, and Ramanarayanan.R, 2012. Probabilistic analysis of storage systems with random sale time depending on production, Int. J. Contemp. Math. Sciences,Vol.7,No.19,943 